\section{Re: ABFM Journal Club's Focus on Critical Appraisal of Full Research Articles is Misplaced}

Dear Drs. Lin, Barry, Ebell, Grad, Shaughnessy, Siwek, and Slawson:

Thank you for your letter, and thank you for raising what is a critical issue for the future of our specialty!

Over the past 30 years, the critical appraisal/evidencebased medicine (EBM) movement has made great strides, with your work playing a crucial role. Patient-oriented evidence that matters (POEMs), a term coined by some of you, is foundational for family physicians' practice of EBM. We appreciate very much your major contributions-providing evidence at the point of care, to journals, to Canadian physicians widely, and increasingly into continuing medical education. As colleagues who were there with you at the beginning, we also celebrate your development of a business model for facilitating the practice of EBM in InfoPOEMs and Essential Evidence Plus.

Our task is different from yours. The actual design of the proposed ABFM National Journal Club ${ }^{1}$ is important to keep in mind. The Journal Club is designed as an optional, not required, continuing certification activity; in addition, Diplomates will be able to choose only those articles they want to study in greater depth. Members of the National Journal Club Committee, who will select the journal club articles and write the accompanying critiques and commentaries, were selected from nominations from all of the specialty organizations and chosen for their clinical experiences, expertise in EBM, and diversity along a number of dimensions. Our literature search process will be explicit and "2 tiered"-building on the foundation you and others have built. In keeping with changes in board certification ${ }^{2}$ and in developing educational theory, our process will include independent assessment and learner engagement and embraces a mastery $^{3}$ approach. All of these features are critical for knowledge retention and application. How many of us have looked up the same information more than once? Like InfoPOEMs, PURLS (Priority Updates from the Research Literature), and similar EBM services, we will endeavor to discuss the findings of individual articles within the context of other important literature and provide clinical direction, modulated by the collective opinion of a geographically diverse committee. Finally, the ABFM National Journal Club, which will identify the top 100 articles for family physicians each year, will be more selective than InfoPOEMS (approximately 250 articles/ year) or NEJM Journal Watch (approximately 400 articles/year). We hope that our curation of the most important literature available will be valuable for Diplomates.

You question the value of reading full text articles. Do we really believe that family physicians should not read full text articles after completing residency? Will medical students choose to go into a field in which that is an expectation? Despite widespread information overload, no other specialty has taken that stance, and physicians report that peer-reviewed articles are viewed as the most useful and influential medical information source. ${ }^{4}$ We believe that we can help make this process more efficient and manageable. More broadly, we believe that knowing the primary literature- the details and limitations, along with the context of other literature-can help customize patient counseling and informed decisionmaking. Practicing family medicine is more than knowing disease management pathways and care algorithms.

Good-quality information can also empower family physicians as they advocate for their patients with subspecialists, payers, and hospital systems across the continuum of care. We believe that this often requires a greater depth of knowledge than summaries can provide. We agree with you that keeping up with methodology seems challenging to many, which is why we will include questions on methodology in our assessments. We do not agree, however, that family physicians should rely on outside experts, however well intentioned and sophisticated, to pronounce $e x$ cathedra on what family physicians need to know and to do.

The ABFM Journal Club is still in the developmental phase. Our pilot this summer will test both feasibilitycan we combine the many components of this project, from article selection to item writing to access to full articles to the information technology backbone?-and also its value to practicing family physicians. We also hope to collaborate with AFMRD and others to promote innovation in teaching and learning using this service. As always, ABFM is committed to continuous quality improvement. We do rigorous evaluation — and act on it.

Thank you once again for your many contributions to this important area. We look forward to the possibility of collaborating with you in the future.

Warren P. Newton, MD, MPH American Board of Family Medicine Lexington, $\mathrm{KY}$ wnewton@theabfm.org

To see this article online, please go to: http://jabfm.org/content/ 34/2/454.full.

\section{References}

1. Quan MA, Newton W, Handler L, Banik E. Empowering family physicians to drive change in practice: plans for the ABFM National Journal Club. Ann Fam Med 2021;19:89-90.

2. Newton WP. How should board certification evolve? J Am Board Fam Med 2020;33:S1-S9.

3. McGahie WC. Mastery learning: it is time for medical education to join the 21st century. Acad Med 2015;90:1438-41.

4. Salinas GD. Trends in physician preferences for and use of sources of medical information in response to questions arising at the point of care: 2009-2013. JCEHP 2014;34:S11-S16.

doi: 10.3122/jabfm.2021.02.210027 